\title{
Estabilidade oxidativa da carne e derivados cárneos com o uso de antioxidantes naturais ou sintéticos em artigos publicados na base de dados da Scielo: revisão de literatura
}

\author{
[Oxidative stability of meat and meat products with the use of natural or synthetic antioxidants in \\ articles published in the Scielo database: Literature review]
}

\section{"Revisão/Review"}

\author{
Eliane Cuaglio Paschoal ${ }^{1 *}$, José Matheus Beltrami ${ }^{1,2}$, Isabela Carvalho dos Santos ${ }^{1}$, \\ Ricardo de Melo Germano ${ }^{1}$, Andréia Assunção Soares ${ }^{1}$, Jaciele Caroline Pereira Dias ${ }^{3}$, \\ André Felipe Berto Almada ${ }^{1}$, Thaís Camaso de Sá ${ }^{1}$, Daniela Dib Gonçalves ${ }^{1}$, \\ Luciana Kazue Otutumi ${ }^{1}$
}

\author{
${ }^{1}$ Programa de Pós-Graduação em Ciência Animal com Ênfase em Produtos Bioativos, Unipar, Umuarama-PR, Brasil. \\ ${ }^{2}$ Taxista PROSUP/CAPES. \\ ${ }^{3}$ Unipar, Umuarama-PR, Brasil. \\ *Autor para correspondência/Corresponding author: E-mail: ely_p_mv@ hotmail.com
}

\begin{abstract}
Resumo
A estabilidade e a qualidade de um alimento armazenado por um período prolongado requerem o uso de antioxidantes tanto sintéticos quanto naturais. No entanto, devido à crescente preocupação em adquirir hábitos alimentares saudáveis e o interesse em consumir produtos alimentícios sem uso de aditivos sintéticos, tornase necessária a pesquisa de fontes de antioxidantes naturais, tendo em vista que a carne é um alimento altamente susceptível à oxidação lipídica em função do teor de ácidos graxos insaturados na sua composição. Sendo assim, o objetivo deste trabalho foi realizar um levantamento de artigos publicados na base de dados da Scielo relacionados ao uso de antioxidantes naturais e sintéticos na carne ou derivados cárneos ou adicionados ração consumida pelos animais. Como estratégia de pesquisa dos dados, utilizou-se as seguintes palavras-chave: "carne e TBARS", "meat and TBARS", "carne e antioxidante", "meat and antioxidant". No total foram identificados 71 artigos oriundos do Brasil, África do Sul, Colômbia, Espanha, Chile, Cuba e Venezuela. No entanto, somente 12 artigos foram analisados, visto que o estudo focou em trabalhos que tivessem sido desenvolvidos com o uso de antioxidantes na carne ou ração dos animais. A utilização de antioxidantes proporciona na sua grande maioria uma melhor estabilidade das propriedades funcionais da carne e seus derivados por meio da diminuição da peroxidação lipídica, promovendo maior tempo de prateleira da carne. No entanto, atenção deve ser dada à legislação que regulamenta o uso dos antioxidantes conforme as suas concentrações limites, permitindo o controle dos processos oxidativos sem comprometer a segurança alimentar.
\end{abstract}

Palavras-chave: estabilidade oxidativa; peroxidação lipídica; produtos cárneos; TBARS.

\begin{abstract}
The stability and quality of a food stored for an extended period requires the use of both synthetic and natural antioxidants. However, due to the growing concern about acquiring healthy eating habits and the interest in consuming food products without the use of synthetic additives, it becomes necessary to search for sources of natural antioxidants, since meat is a food that is highly susceptible to lipid oxidation as a function of the content of unsaturated fatty acids in their composition. Thus, the objective of this work was to make a survey of articles published in the Scielo database related to the use of natural and synthetic antioxidants in the meat or meat products or added to the ration consumed by the animals. As a data search strategy, the following keywords were used: "meat and TBARS" [in Portuguese], "meat and TBARS", "meat and antioxidant"[in Portuguese], "meat and antioxidant". Seventy-one articles were identified from Brazil, South Africa, Colombia, Spain, Chile, Cuba, and Venezuela; however, only 12 articles were analyzed, since the study focused on studies that had been developed with the use of antioxidants in meat or the animals' feed. The use of antioxidants largely provides a better stability of the functional properties of the meat by reducing lipid peroxidation, promoting
\end{abstract}


longer shelf life of the meat. However, attention should be paid to the legislation that regulates the use of antioxidants according to their limiting concentrations, allowing the control of oxidative processes without compromising food safety.

Keywords: oxidative stability; lipid peroxidation; meat products; TBARS.

\section{Introdução}

A carne é um dos alimentos mais importantes na alimentação dos humanos, por ter proteína de alta qualidade, minerais e vitaminas. Contudo, atenção tem sido dada em relação à conservação das suas propriedades funcionais, de tal forma a garantir um produto final de qualidade aos consumidores (Oliveira et al., 2012), principalmente com o uso de substâncias antioxidantes provenientes de vegetais, em função das restrições ao uso de antioxidantes sintéticos (Silva et al., 2010). Dessa forma, estudos relacionados ao uso de antioxidantes naturais na preservação de alimentos têm aumentado (Lee et al., 2005; Estévez e Cava, 2006; Mariutti e Bragagnolo, 2009; Fratianni et al., 2010; Radha Krishnan et al., 2014).

Por outro lado, cabe salientar que a utilização de antioxidantes naturais para retardar a oxidação lipídica nos alimentos não é um conceito novo, visto que por volta de 1980 estudos relacionados com os compostos naturais para preservação ou extensão de vida útil dos alimentos foram feitos, direcionados a substituir ou diminuir o uso dos antioxidantes sintéticos nos alimentos (Brewer, 2011), sendo os antioxidantes naturais adicionados na ração durante a fase de crescimento dos animais ou diretamente na carne ou seus subprodutos (Leão et al., 2017).

Os antioxidantes naturais mais utilizados atualmente são os tocoferóis, os ácidos fenólicos e extratos de plantas como alecrim e sálvia (Ramalho e Jorge, 2006).

No intuito de se avaliar a proteção antioxidante, existem métodos que são utilizados como controle de qualidade de alimentos que contém gordura, no caso de produtos cárneos, tais como o índice de peróxido, ácidos graxos livres, anisidina, kreis, compostos voláteis e ácido tiobarbitúrico (TBA), que geram informações em relação ao estado oxidativo e consequentemente tendências à rancidez do alimento (Osawa et al., 2005).

Dessa forma, o objetivo deste trabalho foi realizar um levantamento de artigos publicados na base de dados da Scielo relacionados ao uso de antioxidantes naturais e sintéticos na carne ou derivados cárneos ou adicionados a ração consumida pelos animais.

\section{Estratégia de busca de artigos na base de dados da SciELO}

Para o desenvolvimento da revisão de literatura, foi utilizado o banco de dados SciELO. O método de pesquisa foi "integrada", conforme descrito no banco de dados.

Como estratégia de pesquisa dos dados, utilizou-se as seguintes palavras-chave: "carne e TBARS", "meat and TBARS", "carne e antioxidante", "meat and antioxidant".

As palavras-chave foram pesquisadas sob os parâmetros "todos os índices" para integrar toda a base de dados. Com base nesse levantamento foram identificados 71 artigos oriundos do Brasil, África do Sul, Colômbia, Espanha, Chile, Cuba e Venezuela.

Todos os artigos publicados até a data da pesquisa (21 de junho de 2016) que retornaram como resultado da busca e que tivesse sido desenvolvido com carne e com o uso de antioxidantes na carne ou ração foram selecionados (Tabela 1).

\section{Seleção de artigos da base de dados da SciELO}

Foram encontrados cinco artigos originais que fizeram uso de antioxidantes sintéticos ou naturais diretamente na carne ou derivados cárneos, sendo dois com galinhas poedeiras brancas e marrons (Trindade et al., 2008; Borba et al., 2012), um com frangos de corte (Fellenberg et al., 2011) um com salsicha tipo Frankfurt oriundo de carne suína e de frangos (Maya et al., 2012) e um com linguiça frescal suína (Stefanello et al., 2015) e que avaliaram a proteção antioxidante medida pela produção do TBARS - Thiobarbituric Acid Reactive Substances (Tabela 2).

Em relação ao uso de antioxidantes sintéticos ou naturais na ração, foram encontrados cinco artigos originais, sendo três em frangos de corte (Freitas et al., 2012; Kim et al., 2015; Lima et al., 2015) e dois em cordeiros (Booyens et al., 2012; Salinas-Rios et al., 2014). Nessas pesquisas, 
foram compilados os resultados dos valores de TBARS da carne no tempo zero (Tabela 3).

Em relação aos artigos de revisão, foram encontrados dois artigos (Velasco e Williams, 2011; Garcia et al., 2012), que foram citados na Tabela 1.

Tabela 1. Artigos selecionados da base de dados da Scielo publicados durante o período de 2008 a 2015 que fizeram uso de antioxidantes naturais ou sintéticos na ração ou carne em relação à proteção antioxidante medida por meio da produção de substâncias reativas ao ácido tiobarbitúrico (TBARS).

\begin{tabular}{lcc}
\hline Nome da revista & Tipo de artigo & Referência \\
\hline Food Science and Technology & Artigo original & Trindade et al. (2008) \\
Chilean Journal of Agricultural Research & Artigo revisão & Velasco e Williams (2011) \\
Revista Brasileira de Ciência Avícola & Artigo original & Fellenberg et al. (2011) \\
South African Journal of Animal Science & Artigo original & Booyens et al. (2012) \\
Revista Brasileira de Saúde e Produção Animal & Artigo original & Borba et al. (2012) \\
Ciência Rural & Artigo revisão & Garcia et al. (2012) \\
Pesquisa Agropecuária Brasileira & Artigo original & Freitas et al. (2012) \\
Revista Facultad Nacional de Agronomía & Artigo original & Maya et al. (2012) \\
Arquivo Brasileiro de Medicina Veterinária e Zootecnia & Artigo original & Salinas-Rios et al. (2014) \\
Animal Science & Artigo original & Kim et al. (2015) \\
Revista Brasileira de Ciência Avícola & Artigo original & Lima et al. (2015) \\
Ciência e Agrotecnologia & Artigo original & Stefanello et al. (2015) \\
\hline
\end{tabular}

Tabela 2. Artigos originais publicados na base de dados da Scielo publicados durante o período de 2008 a 2015 que fizeram uso de antioxidantes naturais ou sintéticos na carne em relação à proteção antioxidante medida por meio da produção de substâncias reativas ao ácido tiobarbitúrico (TBARS).

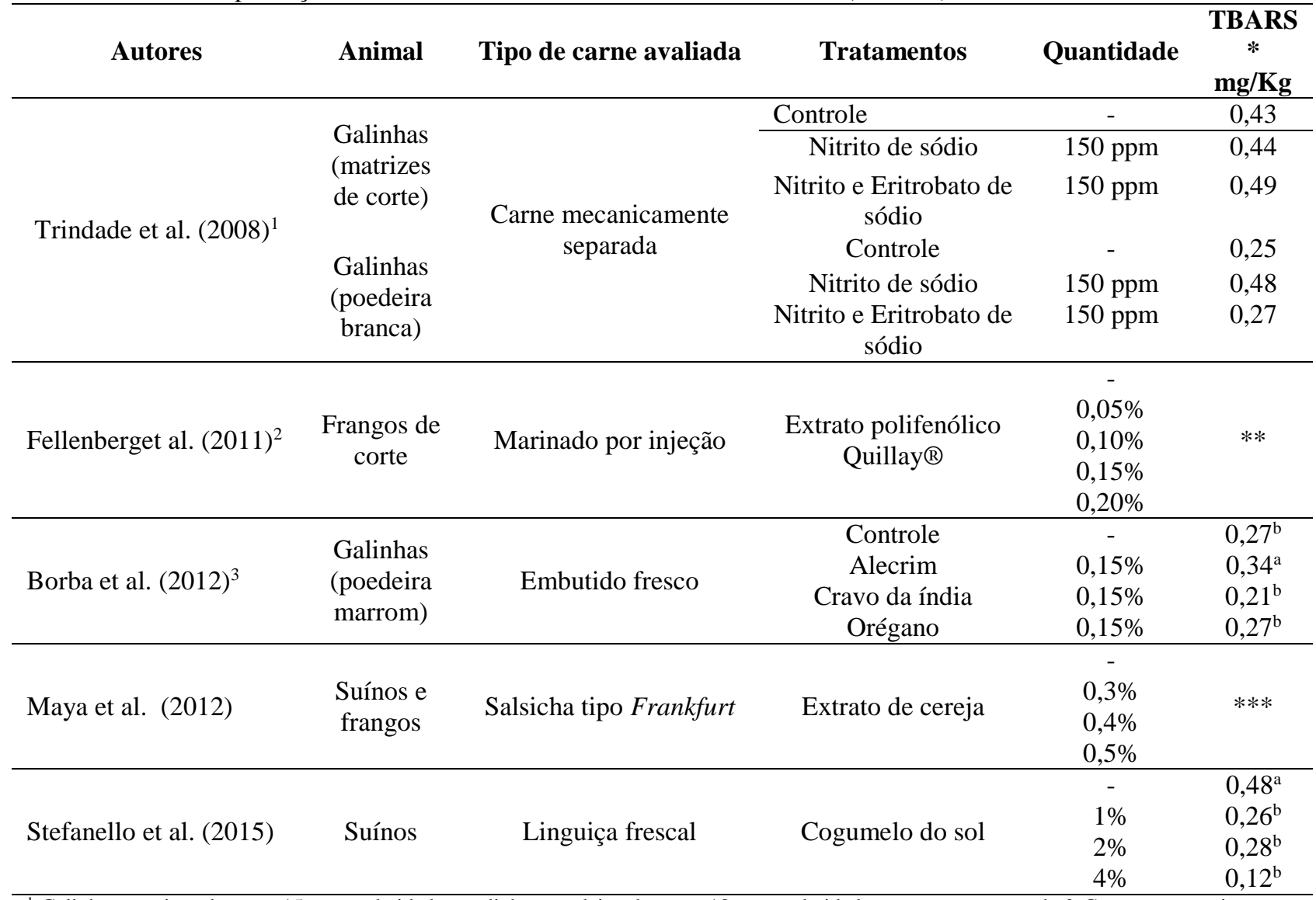

\footnotetext{
${ }^{1}$ Galinhas matrizes de corte 15 meses de idade e galinhas poedeiras brancas 18 meses de idade, com temperatura de $0{ }^{\circ} \mathrm{C}$; carne mecanicamente separada (asas, pernas, dorso e quilha do peito), congelada, $p>0,05 ;{ }^{2}$ Frangos de corte; marinado por injeção (peito), congelado; ${ }^{3}$ Galinhas poedeiras marrom 80 semanas de idade, embutido fresco (carne de peito e pernas); QLPERM. Chile., p<0,05. * medido no dia zero; ** TBARS apresentado com densidade óptica/g, $\mathrm{p}>0,05$; *** TBARS apresentado na forma de gráfico (no dia zero todos os valores foram superiores a $0,5 \mathrm{mg} / \mathrm{Kg}$ ).
}

\section{Discussão dos trabalhos selecionados}

Em relação aos resultados apresentados na Tabela 2 relacionados ao uso de antioxidantes naturais ou sintéticos na carne (carne mecanicamente separada de aves, marinado de frango, embutido fresco de galinha, salsicha tipo Frankfurt e linguiça frescal suína) verificou-se que a proteção antioxidante medida por meio do 
TBARS, no dia zero, foi significativamente diminuída quando da utilização de cogumelo do sol independente da quantidade (1, 2 ou 4\%) (Stefanello et al., 2015) e do uso de $0,15 \%$ de cravo da índia e orégano quando comparado com o grupo controle (Borba et al., 2012). No entanto, não se verificou diferença em relação à proteção antioxidante no dia zero com o uso de nitrito de sódio e eritorbato de sódio (Trindade et al., 2008), extrato de cereja (Maya et al., 2012), extrato polifenólico de Quillay (Fellenberg et al., 2011).

O Quillay (Quillaja saponaria) é uma árvore nativa do Chile e seu extrato polifenólico foi utilizado no trabalho de Fellenberg et al. (2011), em função da literatura tê-lo relacionado como tendo efeito antioxidante. Esse efeito pode estar associado aos componentes do extrato que continha $7,16 \%$ de polifenóis, $1,84 \%$ de tanino e $18,6 \%$ de polissacarídeos.
Segundo Mariutti e Bragagnolo (2007), a capacidade antioxidante dos condimentos, tais como alecrim e orégano avaliados no trabalho de Borba et al. (2012), está relacionada aos seus compostos fenólicos, sendo eles constituintes de uma ampla variedade de classes (fenólicos diterpenos, ácidos fenólicos e fenilpropanóides (Brewer, 2011).

Os trabalhos com o uso de antioxidantes na ração de frangos de corte foram avaliados em três linhagens diferentes (Ross, Cobb e Arbor Acres) (Tabela 3 ) e os valores do TBARS foram medidos em amostras de carne de peito resfriada a $4^{\circ} \mathrm{C}$ (Freitas et al., 2012; Kim et al., 2015; Lima et al., 2015). Já os trabalhos com cordeiros, foram compilados e os resultados do TBARS do lombo resfriado a $4^{\circ} \mathrm{C}$ (Salinas-Rios et al., 2014) ou $5^{\circ} \mathrm{C}$ (Booyens et al., 2012) (Tabela 3).

Tabela 3. Artigos originais publicados na base de dados da Scielo publicados durante o período de 2008 a 2015 que fizeram uso de antioxidantes naturais ou sintéticos na ração de frangos de corte de diferentes linhagens (Ross 308, Cobb 500 e Arbor Acres) e cordeiros e avaliaram a produção de compostos secundários da peroxidação lipídica - TBARS (substâncias reativas ao ácido tiobarbitúrico) na carne refrigerada no dia zero de armazenamento.

\begin{tabular}{|c|c|c|c|c|}
\hline Artigos originais & Animal & Tratamentos & Quantidade & TBARS* mg/Kg \\
\hline \multirow{6}{*}{ Freitas et al. $(2012)^{1}$} & \multirow{6}{*}{$\begin{array}{c}\text { Frangos de } \\
\text { corte } \\
\text { Ross } 308\end{array}$} & Controle & --- & $0,19^{\mathrm{a}}$ \\
\hline & & Butilato de hidroxitolueno (BHT) & 200 ppm & $0,16^{\mathrm{b}}$ \\
\hline & & Extrato da casca da manga (ECAS) & $200 \mathrm{ppm}$ & $0,18^{\mathrm{a}}$ \\
\hline & & Extrato da casca da manga (ECAS) & 400 ppm & $0,19^{\mathrm{a}}$ \\
\hline & & Extrato do caroço da manga (ECAR) & $200 \mathrm{ppm}$ & $0,19^{\mathrm{a}}$ \\
\hline & & Extrato do caroço da manga (ECAR) & $400 \mathrm{ppm}$ & $0,14^{\mathrm{b}}$ \\
\hline \multirow{7}{*}{ Lima et al. $(2015)^{2}$} & \multirow{7}{*}{$\begin{array}{l}\text { Frangos de } \\
\text { corte } \\
\text { Cobb } 500\end{array}$} & Controle & --- & \multirow{7}{*}{$* *$} \\
\hline & & Óleo de resina de Copaíba & 500 ppm & \\
\hline & & Óleo de resina de Copaíba & 900 ppm & \\
\hline & & Óleo de resina de Copaíba & $1300 \mathrm{ppm}$ & \\
\hline & & Óleo de resina de Sucupira & 500 ppm & \\
\hline & & Óleo de resina de Sucupira & $900 \mathrm{ppm}$ & \\
\hline & & Óleo de resina de Sucupira & $1300 \mathrm{ppm}$ & \\
\hline \multirow{3}{*}{ Kim et al. $(2015)^{3}$} & \multirow{3}{*}{$\begin{array}{l}\text { Frangos de } \\
\text { corte } \\
\text { Arbor Acres }\end{array}$} & Controle & - & $0,44^{\mathrm{a}}$ \\
\hline & & Pó da casca de ouriço-do-mar & $1 \%$ & $0,34^{\mathrm{a}}$ \\
\hline & & Aditivos alimentares & $1 \%$ & $0,21^{\mathrm{b}}$ \\
\hline \multirow{2}{*}{ Booyenset al. $(2012)^{4}$} & \multirow{2}{*}{$\begin{array}{l}\text { Cordeiro } \\
\text { Mutton } \\
\text { Merino }\end{array}$} & Antioxidante sintético & $125 \mathrm{~g} / \mathrm{T}$ & $0,13^{\mathrm{a}}$ \\
\hline & & Antioxidante natural & $125 \mathrm{~g} / \mathrm{T}$ & $0,12^{\mathrm{a}}$ \\
\hline \multirow{3}{*}{ Salinas-Rios et al. $(2014)^{5}$} & \multirow{3}{*}{$\begin{array}{l}\text { Cordeiro } \\
\text { Blackbelly }\end{array}$} & Controle & - & \multirow{3}{*}{$* * *$} \\
\hline & & Polpa de café & $8 \%$ & \\
\hline & & Polpa de café & $16 \%$ & \\
\hline
\end{tabular}

* medido no dia zero ${ }^{1}$ carne de peito, $\mathrm{p}<0,05 ;{ }^{2}$ carne de peito, $\mathrm{p}<0,0001 ;{ }^{3}$ carne de peito; aditivos alimentares: Vitagold. Brasil; p<0,05. ${ }^{4}$ Antioxidante sintético: BHT, BHA, etoxiquim e citrato de sódio trihidratado; antioxidante natural: extrato de planta contendo bioflavonóides (catecina e galo-catecina) e pro-antocianidina (que gera as antocianidinas, delfinidina, robinetidina e fisetidina); $\mathrm{p}>0,05 ;{ }^{5}$. Polpa de café foi ensilada com $5 \%$ de melaço, fermentada por dois meses e desidratada ao sol.

** resultados apresentados no formato de figura: Menor valor de TBARS ( $<<0,0001)$ para os tratamentos contendo óleo de copaíba e sucupira;

*** resultados apresentados no formato de gráfico: não houve diferenças entre os tratamentos.

Em relação aos resultados apresentados na Tabela 3, verificou-se redução significativa nos valores TBARS $(\mathrm{mg} / \mathrm{kg})$ da carne de peito no dia zero para os frangos que receberam $400 \mathrm{ppm}$ de extrato de caroço de manga (ECAR) por um período de 42 dias em relação ao grupo controle e 
o grupo suplementado com $200 \mathrm{ppm}$ de ECAR e os suplementados com 200 e 400 ppm de extrato de casca de manga (ECAS) (Freitas et al., 2012), demonstrando efeito positivo em se retardar a oxidação lipídica da carne com a suplementação de 400 ppm de ECAR.

De maneira similar, Lima et al. (2015) verificaram menor valor de TBARS para os animais que receberam a dieta suplementada com óleo de resina de copaíba ou sucupira por um período de 37 dias (Tabela 3) quando comparados com o grupo controle $(\mathrm{p}<0,0001)$.

Em frangos da linhagem Arbor Acres, Kim et al. (2015) (Tabela 3) verificou a redução nos resultados do TBARS da carne do peito para os animais suplementados com $1 \%$ de aditivos alimentares $\left(\right.$ Vitagold $\left.^{\circledR}\right)$ por um período de 28 dias quando comparado com o grupo controle e o grupo tratado com $1 \%$ da casca de ouriço-do-mar. Esse aditivo alimentar ( Vitagold $^{\circledR}$ ) é uma suplementação de vitaminas lipossolúveis e hidrossolúveis recomendado para uso em animais de companhia e de produção, que na sua composição encontra-se: nicotinamida; hidróxido de tolueno butilado (BHT); vitamina $\mathrm{A}$; vitamina $\mathrm{B}_{1}$; vitamina $\mathrm{B}_{2}$; vitamina $\mathrm{B}_{6}$; vitamina $\mathrm{B}_{12}$; vitamina $\mathrm{D}_{3}$ e vitamina E. Segundo Kim et al. (2015), a casca de ouriçodo-mar possui valor nutricional e efeitos farmacêuticos, pois servem como uma boa fonte de proteína, ácidos graxos poliinsaturados, minerais ( $\mathrm{Ca}, \mathrm{Fe}, \mathrm{Mg}$ e Ca), vitaminas (B e C) além de serem utilizados em remédios medicinais. Segundo os autores, o uso do pó do ouriço-do-mar em dietas de animais exige um maior aprofundamento experimental para entender seus mecanismos de ação e efeitos benéficos.

Em cordeiros, ambos os trabalhos (Booyens et al., 2012; Salinas-Rios et al., 2014) (Tabela 3) não demonstraram diferenças significativas nos valores de TBARS na carne de lombo dos animais que foram suplementados com antioxidante sintético (BHT, BHA, etoxiquim e citrato de sódio trihidratado) ou natural (extrato de plantas contendo bioflavonóides - catequinas e galocatequinas) (Booyens et al., 2012) ou receberam polpa de café em percentuais de 8 e $16 \%$ (Salinas-Rios et al., 2014).

Como demonstrado na Tabela 1 , foram identificados dois trabalhos de revisão (Velasco e William, 2011; Garcia et al., 2012). Na revisão de Velasco e Williams (2011), os autores avaliaram a melhoria da qualidade da carne por meio do uso de antioxidantes naturais. Em relação ao assunto revisado, focou-se na avaliação da atividade antioxidante de extratos de plantas por meio da redução da oxidação. Em sua revisão Velasco e Williams (2011), os autores citam uma pesquisa que demonstrou os extratos de sálvia, noz moscada e dente de alho reduziram os níveis de oxidação lipídica $(\mathrm{TBA}<0,5)$ da carne suína quando comparados com o controle. Esses níveis de oxidação lipídica foram determinados pela técnica do TBARS, onde os valores foram expressos como redução do ácido tiobarbitúrico (TBA). Outro resultado importante, encontrado nessa mesma revisão, foi que os óleos essenciais de orégano e sálvia também apresentaram níveis de oxidação lipídica reduzida (TBA $<0,3$ ), quando adicionados à carne bovina e suína durante o período de refrigeração. Esses resultados evidenciaram o potencial antioxidante dessas plantas em reduzir a oxidação lipídica, sendo essa atividade antioxidante atribuída aos seus compostos fenólicos e compostos voláteis presente nos óleos essenciais (Velasco e William, 2011).

Na revisão feita por Garcia et al. (2012), os autores avaliaram o uso dos carotenóides bixina e norbixina extraídos do urucum (Bixa orellana L.) como antioxidantes em produtos cárneos. Segundo os mesmos, apesar do urucum ser autorizado pela legislação brasileira como corante, seu uso em carnes frescais ou congeladas ainda é proibido, no entanto, os resultados de pesquisa revisados por Garcia et al. (2012) avaliando o seu uso como antioxidante mostrou resultados benéficos em hambúrgueres elaborados com peito de frango por meio da redução da rancidez oxidativa e o uso individual da norbixina como redutor de aproximadamente $20 \%$ nos níveis de MDA de salsichas formuladas com mistura de carnes bovina, suína e de frango.

\section{Conclusão}

A utilização de antioxidantes proporciona na sua grande maioria uma melhor estabilidade das propriedades funcionais da carne e derivados cárneos por meio da diminuição da peroxidação lipídica, medido pelo método TBARS, promovendo maior tempo de prateleira da carne. No entanto, a variedade de antioxidantes naturais e de compostos presentes justifica diferenças em seus resultados. Por outro lado, atenção deve ser dada à legislação que regulamenta o uso dos antioxidantes conforme as suas concentrações limites, para que haja um maior controle dos 
processos oxidativos sem que isso comprometa a segurança alimentar.

\section{Conflito de Interesses}

Os autores declaram não existir conflito de interesse.

\section{Referências}

Booyens, K.E.; Einkamerer, O.B.; Hugo, A.; Van Der Merwe; H.J.; Slippers, S.C.; Fair, M.D. Fatty acid composition and oxidative stability of lambs' meat as affected by a bioflavonoid antioxidant and fat sources. South African Journal of Animal Science, 42(5): 483-487, 2012.

Borba, H.; Scatolini-Silva, A.M.; GiampietroGaneco, A.; Boiago, M.M; Souza, P.A. Características físico-químicas e sensoriais de embutido fresco de aves de descarte preparado com diferentes antioxidantes naturais. Revista Brasileira de Saúde e Produção Animal, 13(2): 360-370, 2012.

Brewer, M.S. Natural antioxidants: sources, compounds, mechanisms of action, and potential applications. Comprehensive Reviews in Food Science and Food Safety, 10(4): 221-247, 2011.

Fellenberg, M.A.; Espinoza, A.; Peña, I.; Alarcón, J. Antioxidant and bacteriostatic effects of the addition of extract of Quillay polyphenols (Quillaja saponaria) in the marinade of broiler chicken. Revista Brasileira de Ciência Avícola, 13(1): 71-79, 2011.

Fratianni, F.; De Martino, L.; Melone, A.; De Feo, V.; Coppola, R.; Nazzaro, F. Preservation of chicken breast meat treated with thyme and balm essential oils. Journal of food Science, 75(8): 528-535, 2010.

Freitas, E.R.; Borges, Â.S.; Trevisan, M.T.S.; Watanabe, P.H.; Cunha, A. L.; Pereira, A.L.F.; Abreu, V.K.; Nascimento, G.A.J. Extratos etanólicos da manga como antioxidantes para frangos de corte. Pesquisa Agropecuária Brasileira, 47(8): 1025-1030, 2012.

Estévez, M.; Cava, R. Effectiveness of rosemary essential oil as an inhibitor of lipid and protein oxidation: contradictory effects in different types of frankfurters. Meat Science, 72(2): 348-55, 2006.

Garcia, C.E.R.; Bolognesi, V.J.; Dias, J.F.G.; Miguel, O.G.; Costa, C.K. Carotenoides bixina e norbixina extraídos do urucum (Bixa orellana L.) como antioxidantes em produtos cárneos. Ciência Rural, 42(8): 1510-1517, 2012.

Kim, S.C.; Chung, Y.H.; Chung, T.H; Choi, I.H. Comparative effects of dietary sea urchin shell powder and feed additives on meat quality and fatty acid profiles of broiler breast meat. Acta Scientiarum. Animal Sciences, 37(3): 289294, 2015.

Leão, L.L.; Oliveira, F.S.; Souza, R.S.; Farias, P.K.S.; Da Fonseca, F.S.A.; Martins, E.R.; De Souza, R.M. Uso de antioxidantes naturais em carnes e seus subprodutos. Caderno de Ciências Agrárias, 9(1): 94-100, 2017.

Lee, J.W.; Park, K.S.; Kim, J.G.; Byun, M.W. Combined effects of gamma irradiation and rosemary extract on the shelf-life of a readyto-eat hamburger steak. Radiation Physics and Chemistry, 72(1): 49-56, 2005.

Lima, C.B.; Racanicci, A.M.C.; Oliveira, G.R.; Migotto, D.L.; Amador, S.A.; Souza, T.C.; Tanure, C.B.G.S.; Vieira, A. Effects of the dietary supplementation of sucupira (Pterodon Emarginatus Vog.) and copaiba (Copaifera Langsdorffii) resinoils on chicken breast and thigh meat quality and oxidative stability. Revista Brasileira de Ciência Avícola, 17(SPE): 47-55, 2015.

Mariutti, L.R.B.; Bragagnolo, N.A. oxidação lipídica em carne de frango e o impacto da adição de sálvia (Salvia officinalis, L.) e de alho (Allium sativum, L.) como antioxidantes naturais. Revista do Instituto Adolfo Lutz, 68(1): 1-11, 2009.

Maya, Y.L.I.; Molina, D.A.R.; Vargas, J.H.L. Efecto de la inclusión de un extracto de cereza (Prunus avium L.) sobre el estado de oxidación y las características fisicoquímicas y sensoriales de salchichas tipo Frankfurt. Revista Facultad Nacional de Agronomia Medellin, 65(1): 6541-6552, 2012.

Oliveira, R.R; Lage, M.E; Silveira-Neto, O.J; Sales, M.C. Antioxidantes naturais em produtos cárneos. Publicações em Medicina Veterinária e Zootecnia, 6(10), 2012.

Osawa, C.C.; Felício, P.E.; Gonçalves, L.A.G. Teste de TBA aplicado a carnes e derivados: métodos tradicionais, modificados e alternativos. Química Nova, 28(4): 655-663, 2005.

Ramalho, V.C.; Jorge, N. Antioxidantes utilizados em óleos, gorduras e alimentos gordurosos. Química Nova, 29(4): 755-760, 2006. 
Radha Krishnan, K.; Babuskin, S.; Babu, P.A.S.; Sasikala, M.; Sabina, K.; Archana, G.; Sivarajan, M.; Sukumar, M. Antimicrobial and antioxidant effects of spice extracts on the shelf life extension of raw chicken meat. International journal of food microbiology, 171: 32-40, 2014.

Salinas-Rios, T.; Sánchez-Torres-Esqueda, M.T.; Hernández-Bautista, J.; Díaz-Cruz, A.; NavaCuellar, C.; Ortega-Cerrilla, M.E.; CorderoMora, J.L.; Vaquera-Huerta, H.; Velasco, J.L.F. Carcass characteristics, physicochemical changes and oxidative stress indicators of meat from sheep fed diets with coffee pulp. Arquivo Brasileiro de Medicina Veterinária e Zootecnia, 66(6): 1901-1908, 2014.

Silva, M.L.C.; Costa, R.S.; Santana, A.S.; Koblitz, M.G.B. Compostos fenólicos, carotenóides e atividade antioxidante em produtos vegetais. Ciências Agrárias, 31(3): 669-682, 2010.

Stefanello, F.S.; Cavalheiro, C.P.; Ludtke, F.L.; Silva, M.S.; Fries, L.L.M.; Kubota, E.H. Oxidative and microbiological stability of fresh pork sausage with added sun mushroom powder. Ciência e Agrotecnologia, 39(4): 381-389, 2015.

Trindade, M.A.; Nunes, T.P.; Contreras-Castillo, C.J.; Felício, P.E. Estabilidade oxidativa e microbiológica em carne de galinha mecanicamente separada e adicionada de antioxidantes durante período de armazenamento a-18 $\quad$ C. Ciência e Tecnologia de Alimentos, 28(1): 160-168, 2008.

Velasco, V.; Williams, P. Improving meat quality through natural antioxidants. Chilean Journal of Agricultural Research, 71(2): 313-322, 2011. 